\title{
Bifunctional iRGD-anti-CD3 enhances antitumor potency of $T$ cells by facilitating tumor infiltration and $\mathrm{T}$ - cell activation
}

\author{
Shujuan Zhou (D) , Fanyan Meng, Shiyao Du, Hanqing Qian, Naiqing Ding, \\ Huizi Sha, Mei Zhu, Xiaoxiao Yu, Lifeng Wang, Baorui Liu, Jia Wei
}

To cite: Zhou S, Meng F, Du S, et al. Bifunctional iRGD-anti-CD3 enhances antitumor potency of $\mathrm{T}$ cells by facilitating tumor infiltration and T-cell activation. Journal for ImmunoTherapy of Cancer 2021;9:e001925. doi:10.1136/ jitc-2020-001925

- Additional supplemental material is published online only. To view, please visit the journal online (http://dx.doi.org/10. 1136/jitc-2020-001925).

SZ and FM contributed equally. Accepted 02 April 2021

Check for updates

(C) Author(s) (or their employer(s)) 2021. Re-use permitted under CC BY-NC. No commercial re-use. See rights and permissions. Published by BMJ.

The Comprehensive Cancer Centre of Drum Tower Hospital, Medical School of Nanjing University and Clinical Cancer Institute of Nanjing University, Nanjing, China

\section{Correspondence to}

Professor Jia Wei;

jiawei99@nju.edu.cn

Dr Baorui Liu;

baoruiliu@nju.edu.cn

\section{ABSTRACT}

Background Poor infiltration and limited activation of transferred $T$ cells are fundamental factors impeding the development of adoptive cell immunotherapy in solid tumors. A tumor-penetrating peptide iRGD has been widely used to deliver drugs deep into tumor tissues. CD3-targeting bispecific antibodies represent a promising immunotherapy which recruits and activates $T$ cells. Methods T-cell penetration was demonstrated in tumor spheroids using confocal microscope, and in xenografted tumors by histology and in vivo real-time fluorescence imaging. Activation and cytotoxicity of T cells were assessed by flow cytometry and confocal microscope. Bioluminescence imaging was used to evaluate in vivo antitumor effects, and transmission electron microscopy was used for mechanistic studies.

Results We generated a novel bifunctional agent iRGDanti-CD3 which could immobilize iRGD on the surface of $T$ cells through $\mathrm{CD} 3$ engaging. We found that iRGD-antiCD3 modification not only facilitated T-cell infiltration in 3D tumor spheroids and xenografted tumor nodules but also induced T-cell activation and cytotoxicity against target cancer cells. T cells modified with iRGD-anti-CD3 significantly inhibited tumor growth and prolonged survival in several xenograft mouse models, which was further enhanced by the combination of programmed cell death protein 1 (PD-1) blockade. Mechanistic studies revealed that iRGD-anti-CD3 initiated a transport pathway called vesiculovacuolar organelles in the endothelial cytoplasm to promote T-cell extravasation.

Conclusion Altogether, we show that iRGD-anti-CD3 modification is an innovative and bifunctional strategy to overcome major bottlenecks in adoptive cell therapy. Moreover, we demonstrate that combination with PD-1 blockade can further improve antitumor efficacy of iRGDanti-CD3-modified T cells.

\section{BACKGROUND}

Cancer immunotherapies have led to a paradigm shift in cancer treatment over the last decade. Despite the remarkable success in certain tumor types such as advanced melanoma, the majority of patients with solid tumors remain unresponsive. Multiple lines of evidence indicate that abundant T-cell infiltration predicts responses to immunotherapies and corelates with favorable prognosis. ${ }^{12}$ However, there are diverse mechanisms by which solid tumors can disturb T-cell trafficking, including mismatching of chemokines and their receptors, aberrant adhesion molecules expression and dysregulated vascular permeability. ${ }^{3-5}$ A preclinical study has shown that only an extremely small fraction (less than 1\%) of transferred $\mathrm{T}$ cells could actually infiltrate into tumor tissues. ${ }^{6}$ Thus, enhancing T-cell infiltration is critical for broadening success of immunotherapies in solid tumors.

The tumor-penetrating peptide iRGD (sequence: CRGDKGPDC) has been extensively demonstrated to improve penetration of anticancer drugs, including chemotherapeutics, monoclonal antibodies and nanoparticles into tumors since identified in 2009. ${ }^{7-10}$ iRGD follows a multistep and tumor-specific mechanism; first, it binds to integrins $\alpha v \beta 3 / \beta 5$ elevated in the tumor vascular endothelium and various tumor cells, and is then proteolytically cleaved to expose the C-end rule motif, which binds to neuropilin-1 (NRP-1) and triggers downstream penetration pathways. ${ }^{11}$ Recently, our team has reported that iRGD could also facilitate tumor infiltration of transferred $\mathrm{T}$ cells. ${ }^{12}$ Specifically, iRGD was connected to 1,2-distearoyl-sn-glycero-3 -phospho-ethanolamine-polyethylene glycol (DSPE-PEG); the latter inserted into lipid bilayer membrane of $\mathrm{T}$ cells, thereby immobilizing iRGD on the T-cell surface. These iRGD-modified $\mathrm{T}$ cells acquired significantly increased capacity to penetrate deep into tumor tissues. However, this strategy still has much room to improve. DSPE-PEG could not provide any stimulatory signals to activate $\mathrm{T}$ cells, which limited T-cell killing efficacy when they arrive at the tumor site. Moreover, 
despite no significant impairments of $\mathrm{T}$ cells observed within 24 hours after exposing to DSPE-PEG, long-term effects of DSPE-PEG on stability and permeability of cell membrane, cell vitality and effector functions remain unclear. ${ }^{13}$ Therefore, we seek to find an optimal iRGD modification strategy to overcome the aforementioned shortcomings.

Bispecific T-cell engagers (BiTEs) then came into our sight as one of them, namely, blinatumomab, was approved by the Food and Drug Administration and European Medicines Agency for clinical use. BiTEs consist of two monovalent single-chain variable fragments (scFvs) originating from two separate antibodies specific for surface antigens on tumor cells and CD $3 \varepsilon$ on T cells. Once bound to tumor antigens, BiTEs induce CD3 clustering on T cells and thereafter lead to T cell activation, proliferation and redirected lysis against target cancer cells. ${ }^{14-16}$ Based on these, we hypothesise that using the anti-CD3 scFv to bridge T cells and iRGD might not only endow $\mathrm{T}$ cells with superior penetration ability but also activate tumor-infiltrating $\mathrm{T}$ cells to unleash more antitumor potential. What's more, the anti-CD3 scFv connects to $\mathrm{T}$ cells through binding of CD3 $\varepsilon$ in the TCR complex without disruption on the T-cell membrane, thereby circumventing uncertainties associated with DSPE-PEG.

In this study, we constructed a novel recombinant agent iRGD-anti-CD3. Using this agent, iRGD could be modified on the T-cell surface via the anti-CD3 scFv. We examined tumor penetration, activation, and cytotoxicity of $\mathrm{T}$ cells modified with iRGD-anti-CD3. We also demonstrated in vivo antitumor efficacy of these modified $\mathrm{T}$ cells as well as a further combination with PD-1 blockade. Moreover, we uncovered ultrastructural mechanisms on how iRGDanti-CD3 promoted T-cell penetration.

\section{METHODS \\ Cell lines}

Human gastric cancer cell lines MKN45, HGC27, and MGC803, and human colorectal cancer cell line Lovo were purchased from the Cell Bank of Shanghai Institute of Biochemistry and Cell Biology. All cell lines were cultured in Roswell Park Memorial Institute (RPMI) 1640 supplemented with $10 \%$ fetal calf serum, $100 \mathrm{U} / \mathrm{mL}$ penicillin and $100 \mu \mathrm{g} / \mathrm{mL}$ streptomycin at $37^{\circ} \mathrm{C}$ and $5 \% \mathrm{CO}_{2}$. Cell identities were confirmed by phenotype or genotype. Cells were tested for Mycoplasma and only Mycoplasma-free cells were used.

\section{Isolation and culture of human T cells}

Peripheral blood mononuclear cells (PBMCs) from healthy donors were obtained by Ficoll density centrifugation and suspended in AIM-V medium (Gibco, USA) supplemented with $10 \%$ fetal bovine serum. For T-cell activation, PBMCs were cultured by adherence for 2 hours and non-adherent $\mathrm{T}$ cells were activated with 1000 $\mathrm{U} / \mathrm{mL}$ interferon gamma (IFN- $\gamma$ ) (PeproTech, USA) on day 1 and $50 \mathrm{ng} / \mathrm{mL}$ OKT3 (eBioscience, USA) on day
2. Activated cells were expanded in complete medium containing 90\% AIM-V (Gibico, USA), $10 \%$ fetal bovine serum (FBS) serum (Gibico, USA), $300 \mathrm{U} / \mathrm{mL}$ IL-2 (Peprotech, USA), and $50 \mathrm{ng} / \mathrm{mL}$ IL-15 (PeproTech, USA).

\section{Generation of iRGD-anti-CD3}

iRGD-anti-CD3 was designed in the following orientation: His tag-anti-CD3VH-anti-CD3VL-iRGD. The DNA fragments were synthesized, cloned into bacterial expression vector pET28a by Genscript (Nanjing, China), and confirmed by enzyme digestion and DNA sequencing. Proteins were expressed in Escherichia coli BL21 (DE3) after induction by isopropyl $\beta$-D-1-thiogalactopyranoside. Bacterial cultures were harvested by centrifugation, resuspended, and disrupted by sonication. Inclusion bodies from the precipitation of ultrasonic lysate were solubilized in $8 \mathrm{M}$ urea and dialyzed against at least 50-fold volume of renaturation buffer ( $6 \mathrm{M}-4 \mathrm{M}-2 \mathrm{M}$ urea, 0.2 $\mathrm{mM} / 1 \mathrm{mM}$ oxidized/reduced glutathione, $1 \%$ glycine and $1 \mathrm{mM}$ EDTA, $\mathrm{pH} 7.4-7.5$ ) at $4^{\circ} \mathrm{C}$ for 6 hours with rapid mixing, followed by dialysis with only phosphate buffered saline (PBS) for 24-48 hours at $4^{\circ} \mathrm{C}$. The products were subjected to $12 \%$ sodium dodecyl sulfate-polyacrylamide gel electrophoresis (SDS-PAGE) analysis for confirmation. Refolded iRGD-anti-CD3 was purified using an AKTA system HisTrap HP column (GE healthcare, CT, USA) according to the manufacturer's instructions. The eluted solutions were dialyzed against PBS, sterile-filtered $(0.22 \mu \mathrm{m})$, and confirmed by western blot (WB) analysis using an anti-His antibody (Genscript, A00186-100). Protein concentrations were determined using a BCA assay kit (Thermo Fisher Scientific, Massachusetts, USA).

\section{Flow cytometry analysis}

For dual binding assays, $1 \times 10^{5}$ MKN 45 or $1 \times 10^{5}$ PBMCs were incubated with $1 \mu \mathrm{g}$ iRGD-anti-CD3 or control reagents for $30 \mathrm{~min}$ at $4^{\circ} \mathrm{C}$, washed, and then incubated with a secondary mAb against His-tag conjugated to $\mathrm{PE}$ (GG11-8F3.5.1, Miltenyi Biotec GmbH). Expression of iRGD receptors on MGC803 and LOVO was measured by

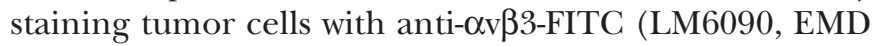
Millipore), anti- $\alpha \mathrm{v} \beta 5$-FITC (P1F6, EMD Millipore), and anti-NRP-1-PE (AD5-17F6, Miltenyi Biotec GmbH). All samples tested were suspended in flow cytometry staining buffer (FACS) buffer and stained with the indicated antibodies for $30 \mathrm{~min}$ at $4^{\circ} \mathrm{C}$ in the dark, washed twice, and resuspended in FACS buffer before analysis. Flow cytometry data were collected on Accuri C6 (BD Bioscience, NJ, USA) and analyzed with FlowJo V.10.4 software. For cell sorting, activated $\mathrm{T}$ cells were incubated with antiCD3-PC7 (5 $\mu \mathrm{L} / 107$ cells, MHCD0312; BD Bioscience), anti-CD4-FITC ( $5 \mu \mathrm{L} / 107$ cells, MHCD0401-4; BD Bioscience), and anti-CD8-APC ( $5 \mu \mathrm{L} / 107$ cells, MHCD0805; BD Bioscience) for $40 \mathrm{~min}$ at $4^{\circ} \mathrm{C}$ and washed. $\mathrm{CD} 3^{+} \mathrm{CD} 4^{+}$ and $\mathrm{CD}^{+} \mathrm{CD}^{+} \mathrm{T}$ cells were negatively isolated on MoFlo XDP Cell Sorter (Beckman Coulter). 


\section{Surface plasmon resonance (SPR)}

SPR experiments were performed on Biacore $8 \mathrm{~K}$ (GE Healthcare) with the CM5 sensor chip. iRGD-anti-CD3 was diluted in acetate 4.5 to $20 \mu \mathrm{g} / \mathrm{mL}$ and injected into the channels of the CM5 chip at a flow rate of $10 \mu \mathrm{L} / \mathrm{min}$ for $400 \mathrm{~s}$. The human CD3 was serially diluted by threefold in running buffer, with the start concentration of $100 \mathrm{nM}$, $90 \mathrm{~s}$ association, $300 \mathrm{~s}$ dissociation, and extra wash using running buffer. Non-specific binding was determined on the chip without antigen. Binding kinetics were analyzed by using Biacore $8 \mathrm{~K}$ software.

\section{iRGD-anti-CD3 internalization}

Modified T cells were harvested from culture medium or serum at the indicated time points. T cells were then fixed with $4 \%$ paraformaldehyde solution (PFA), permeated with $0.5 \%$ TritonX-100, blocked with $1 \%$ bovine serum albumin (BSA) in PBS, and further incubated with an anti-6X His tag antibody (EPR20547, Abcam) for 16 hours at $4^{\circ} \mathrm{C}$, following $4^{\prime}, 6$-diamidino-2-phenylindole (DAPI) staining for $10 \mathrm{~min}$ at room temperature. Confocal microscopy was carried out with a $\times 60$ oil-immersion objective lens using Leica TCS SP5 microscope following standard protocol. Images were analyzed using ImageJ software V.15.1 n.

\section{T-cell penetration in multicellular spheroids (MCSs)}

Spheroids were generated as previously described and monitored with a light microscope. The uniform and compact tumor spheroids were selected for studies when their size reached about $500 \mu \mathrm{m}$. PBMCs were first labeled with carboxyfluorescein succinimidyl ester (CFSE) (Abcam, Cambridge, UK). Then, PBMCs modified with iRGD-anti-CD3 or control reagents $(1 \mu \mathrm{g}$ modification reagent for every $1 \times 10^{5} \mathrm{~T}$ cells), or codelivered with free iRGD were added into the MCSs at an effector-to-target (E:T) ratio of 5:1. After incubation for 24 hours at $37^{\circ} \mathrm{C}$, the spheroids were washed, fixed in $4 \%$ paraformaldehyde, and imaged using a ZEN 710 confocal microscope (Zeiss, Jena, Germany). Images were acquired at the midheight of the spheroids and surface plots were obtained using ImageJ software.

\section{T-cell activation and cytotoxicity assays}

Freshly isolated PBMCs were modified with iRGDanti-CD3 or control reagents $(1 \mu \mathrm{g}$ modification reagent for every $1 \times 10^{5} \mathrm{~T}$ cell) and cocultured with MKN45 at an E:T ratio of 20:1. After incubation for 48 hours at $37^{\circ} \mathrm{C}$, T-cell activation was assessed by flow cytometry analysis of anti-CD4-FITC (RPA-T4, BD Bioscience), anti-CD8Percp-Cy5.5 (RPA-T8, BD Bioscience), anti-CD69-PE (MA1-10276, Invitrogen), and anti-CD25-APC (M-A251, BD Bioscience). The supernatant fluids were harvested for cytokine quantification using the BD CBA Human Th1/ Th2 Cytokine Kit (BD Bioscience, NZ, USA). For cytotoxicity assays, modified PBMCs were incubated with MKN45 at different E:T ratios for 24 hours. The cytotoxicity was measured using the lactate dehydrogenase detection kit
(Roche, Basel, Switzerland) according to the manufacturer's instructions. Tumor cell lysis was also visualized using a light microscope (Leica, Wetzlar, Germany).

For cytotoxicity assay in MCSs, PBMCs modified with iRGD-anti-CD3 or control reagents $(1 \mu \mathrm{g}$ modification reagent for every $1 \times 10^{5} \mathrm{~T}$ cells), or codelivered with free iRGD were added into the MCSs at an E:T ratio of 20:1. After incubation for 24 hours at $37^{\circ} \mathrm{C}$, the spheroids were washed, stained using a Viability/Cytotoxicity Kit (Biotium, California, USA), and fixed. Images were taken using a ZEN 880 confocal microscope (Zeiss) as Z-stack projects scanning from the top to the middle of the MCSs with $5 \mu \mathrm{m}$ intervals and then presented as maximum intensity projections. Surface displays (2.5 D) were obtained using Zeiss image processing software. To quantification live/dead cells, the total cell area of each dye was measured using ImageJ software V.15.1 n.

\section{Xenograft mouse models}

Investigators were not blinded for the animal studies. All efforts were made to minimize the number of animals used and their suffering. Mice were randomized on the basis of age and weight. For the peritoneal metastasis tumor models, 6-8 week old male BALB/c nude mice were injected intraperitoneally with $10^{6}$ MKN45 cells, $5 \times 10^{6}$ MGC803 cells, or $10^{6}$ LOVO cells. For the subcutaneous tumor model, $6-8$ week old male BALB/c nude mice were injected subcutaneously with $10^{6} \mathrm{MKN} 45$ cells.

\section{In vivo real time near-infrared fluorescence imaging}

Near-infrared imaging was used to quantitatively localize $\mathrm{T}$ cells. $\mathrm{T}$ cells were first labeled with the near-infrared fluorescent probe DiR (Bridgen, Beijing, China). T cells $\left(1 \times 10^{7}\right)$ modified with $100 \mu \mathrm{g}$ iRGD-anti-CD3 or control reagents, or codelivered with $3 \mu \mathrm{g}$ free iRGD were injected intraperitoneally or intravenously into MKN45 tumorbearing mice. The mice were anesthetized and scanned using CRi Maestro In Vivo Imaging System (Cambridge Research \& Instrumentation, Massachusetts, USA) at indicated time points post-T-cell administration. For resected tissue imaging, the mice were sacrificed under deep anesthesia. Tumors and main organs, including heart, liver, spleen, lung, kidney, and intestine, were excised and imaged.

\section{Immunofluorescence confocal imaging}

T cells were labeled with CFSE (Abcam, Cambridge, UK). $\mathrm{T}$ cells $\left(1 \times 10^{7}\right)$ modified with $100 \mu \mathrm{g}$ iRGD-anti-CD3 or control reagents, or codelivered with $3 \mu \mathrm{g}$ free iRGD were injected into subcutaneous tumor-bearing mice via the tail vein. Tumors were harvested at 24 hours after injection and processed for immunostaining. Frozen sections were stained with an anti-CD31 antibody (550274, BD Pharmingen) followed by a Cy3-conjugated secondary antibody (A10522, Life Technologies). After washing with PBS, the sections were mounted with DAPI (Beyotime, Shanghai, China) and imaged at $\times 200$ magnification. 


\section{Immunohistochemistry analysis}

To evaluate T-cell infiltration in the peritoneal metastasis tumor model, $1 \times 10^{7} \mathrm{~T}$ cells modified with $100 \mu \mathrm{g}$ iRGDanti-CD3 or control reagents, or codelivered with $3 \mu \mathrm{g}$ free iRGD were injected intraperitoneally into tumorbearing mice. Tumors were collected at 6 hours after injection, fixed in $10 \%$ neutral-buffered formalin, and embedded in paraffin. Infiltrated $\mathrm{T}$ cells were detected by an anti-CD3 mAb (SP7, Abcam). For programmed cell death 1 ligand 1 (PD-L1) and PD-1 detection, mice bearing MKN45 peritoneal metastasis tumors were randomized into two groups. Two weeks after tumor implantation (on day 15 ), $1 \times 10^{7} \mathrm{~T}$ cells modified with $100 \mu \mathrm{g}$ iRGD-anti-CD3 or control reagents, or codelivered with $3 \mu \mathrm{g}$ free iRGD were given intraperitoneally every other day for 5 days. One week later, peritoneal tumors were excised and processed for PD-L1 (SP142, Abcam) and PD-1 staining (EPR4877(2), Abcam).

\section{In vivo antitumor efficacy and safety}

In the MKN45-luc model, peritoneal tumor-bearing mice were randomized into seven groups. Two weeks after tumor implantation (on day 15 ), $1 \times 10^{7} \mathrm{~T}$ cells modified with $100 \mu \mathrm{g}$ iRGD-anti-CD3 or control reagents, or codelivered with $3 \mu \mathrm{g}$ free iRGD were given intraperitoneally every other day for 5 days. PD-1 blockade (250 $\mu \mathrm{g}$ ) was administered intraperitoneally every other day for a total of three injections. Tumor burden was monitored before (day 14), 1 week (day 21), and 2 weeks (day 28) after the start of treatment using the IVIS Lumina III system (PerkinElmer, Massachusetts, USA). In the MGC803 model, treatment timelines, doses, and administration methods followed that of MKN45-luc. On day 28, mice were sacrificed and tumor nodules were weighted. In the LOVO model, treatment began 1 week after tumor induction, with the same treatment schedule as in the MKN45-luc model. On day 21, grades of abdominal distention were recorded in each group. Both tumors and ascites were collected for quantitative analysis. In the subcutaneous mouse model, treatment started when tumor volumes reached approximately $100 \mathrm{~mm}^{3}$. T cells $\left(1 \times 10^{7}\right)$ modified with $100 \mu \mathrm{g}$ iRGDanti-CD3 or control reagents, or codelivered with $3 \mu \mathrm{g}$ free iRGD were given intravenously every other day for 5 days. PD-1 blockade $(250 \mu \mathrm{g})$ was administered every other day for a total of three injections. Tumor size was inspected every 3 days, calculated by the formula length $\times$ width $2 \times 0.5$. For safety studies, major organs were harvested, fixed in $4 \%$ paraformaldehyde, sectioned, and stained with $\mathrm{H} \& \mathrm{E}$.

\section{Transmission electron microscopy (TEM) imaging}

iRGD-anti-CD3 (100 $\mu \mathrm{g}$ ) or RGD-anti-CD3 were injected in mice bearing MKN45 subcutaneous tumors. Tumors were harvested 24 hours after injection, washed in PBS, and immediately fixed with $2.5 \%$ glutaraldehyde at $4^{\circ} \mathrm{C}$. Further process was performed by the electron microscopy services department at Servicebio
(Wuhan, China). Images were obtained and analyzed with HT7700 TEM (Hitachi, Tokyo, Japan). To calculate vesicle density, at least five regions of interest in each group were measured.

\section{Statistical analysis}

GraphPad Prism V.7.0 (GraphPad Software, San Diego, California, USA) was used for all statistical analyses. Variance was similar between the groups that were compared statistically. No statistical methods were used to predetermine sample size. Data are presented as mean \pm SEM. Student's t-test was used to determine the significance between groups. A p value of $<0.05$ was considered statistically significant.

\section{RESULTS}

\section{Generation and characterization of iRGD-anti-CD3}

To construct iRGD-anti-CD3, scFv of the anti-CD3 antibody at the N-terminus was fused to iRGD at the C-terminus by a flexible glycine-serine (G4S) linker. A His tag was inserted upstream of the scFv for purification and detection (figure 1A). The resulting protein was successfully induced in E. coli BL21 and refolded from inclusion bodies, migrating as a major band at approximately $35 \mathrm{kDa}$ in SDS-PAGE (online supplemental figure S1). Purification of iRGD-anti-CD3 using metal affinity chromatography showed a distinct peak in the linear elution profiles (figure 1B). Purified fractions were collected and verified by WB analysis, further confirming the expected molecular size (figure 1C). Two other control antibodies were constructed by replacing iRGD with RGD or $\mathrm{CG}_{7} \mathrm{C}$. The RGD peptide could interact with $\alpha v \beta 3 / \beta 5$ integrins but lacked affinity to NRP-1, while $\mathrm{CG}_{7} \mathrm{C}$ showed no binding to tumor blood vessels or tumors and served as a negative control. ${ }^{7}$

We first evaluated the dual binding of purified iRGDanti-CD3 to target cells (figure 1D,E). Flow cytometry revealed that iRGD-anti-CD3 efficiently engaged both MKN45 cells, which have been identified to express receptors for iRGD, and human CD3 T cells. ${ }^{12}$ RGDanti-CD3 exhibited similar binding profiles, while $\mathrm{CG}_{7} \mathrm{C}$ anti-CD3 was reactive with $\mathrm{T}$ cells but not tumor cells. We next studied the dose-response of T-cell binding of iRGD-anti-CD3. iRGD-anti-CD3 (1 $\mu \mathrm{g}$ ) led to 100\% coating of $10^{5}$ CD3 $\mathrm{T}$ cells (figure $1 \mathrm{~F}$ ). SPR analysis of iRGD-anti-CD3 binding against CD3 $\varepsilon$ yielded a $\mathrm{Kd}$ of

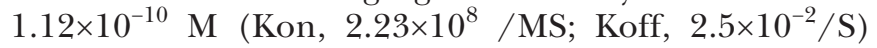
(online supplemental figure S2A).

We then explored binding dynamics of iRGD-anti-CD3 in culture medium and in serum in vivo. By flow cytometry, the fluorescence intensity of iRGD-anti-CD3 on modified $\mathrm{T}$ cells declined to 50\% 30 hours after culturing and 24 hours after transfusion (figure 1G,H). Confocal images revealed that loss of iRGD-anti-CD3 on T-cell surface was due to both antibody internalization and dissociation. iRGD-anti-CD3 exhibited intracellular accumulation at 24 hours and then filled the entire 
A

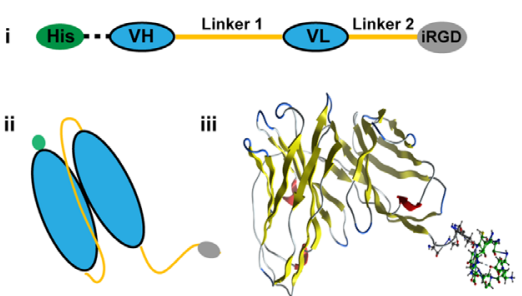

D

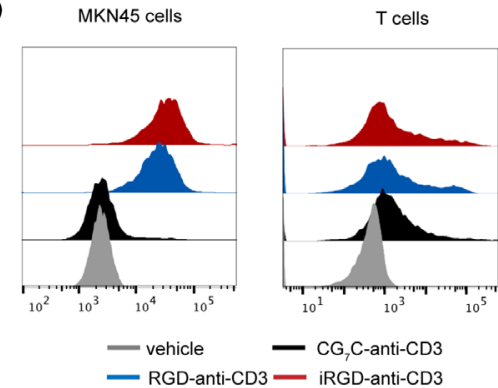

B mAU

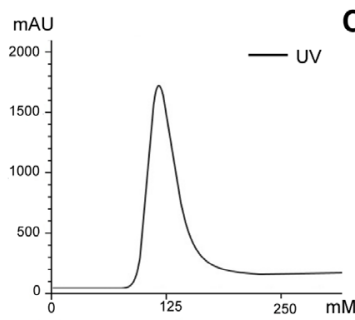

C $(\mathrm{kDa}) \quad 1 \quad 2$

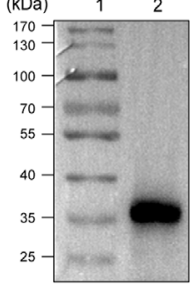

E
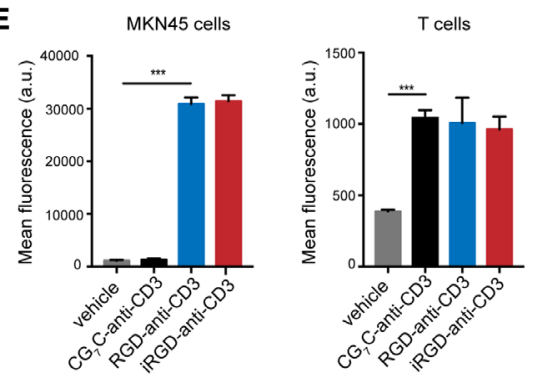

$\mathbf{F}$
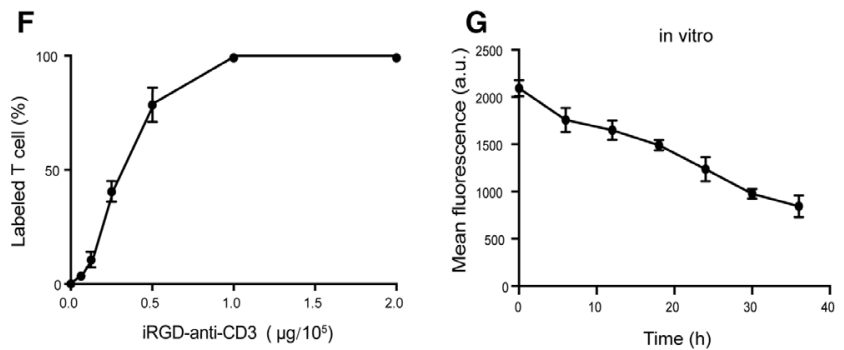

H

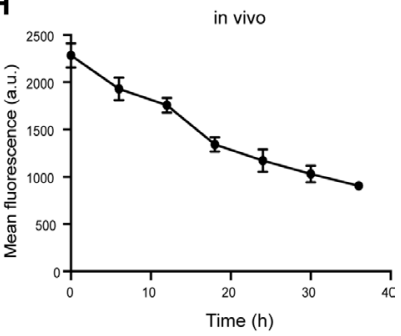
$\mathrm{Oh}$ $12 \mathrm{~h}$ $24 \mathrm{~h}$ $36 \mathrm{~h}$
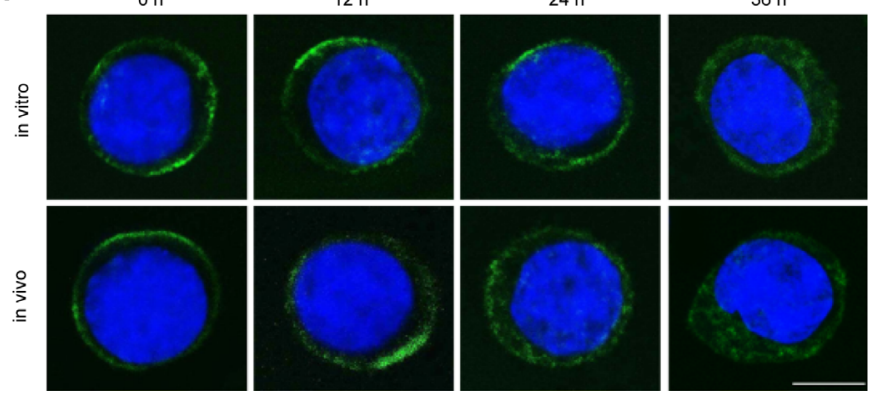

Figure 1 Generation and binding properties of iRGD-anti-CD3. (A) (i) The design of DNA fragments coding iRGD-anti-CD3. Linker 1, G4S ×3; linker 2, G4s; (ii) schematic illustration of iRGD-anti-CD3 structure; (iii) and molecular dynamics simulation using the molecular operating environment 2014 (Montreal, Quebec, Canada). (B) Purification profile of refolded iRGD-anti-CD3. UV, ultraviolet. (C) Eluted fractions were identified by western blot analysis. Lane 1, protein ladder; lane 2, purified iRGD-antiCD3. (D) Flow cytometry results showing bindings of different reagents to MKN45 and PBMCs. (E) Mean fluorescence intensity on MKN45 and PBMCs in (D). Data are represented as mean \pm SEM, $n=3$. Student's t-test, ${ }^{* \star *} p<0.001$. (F) Analysis of the percentage of T cells bound with iRGD-anti-CD3 using flow cytometry. (G) T cells modified with iRGD-anti-CD3 were cultured in vitro for 12-36 hours, and averaged fluorescence intensities of T cells were analyzed by flow cytometry. $(H)$ Mice were injected with T cells modified with iRGD-anti-CD3 via tail vein, and then blood was drawn 12-36 hours after injection. Averaged fluorescence intensities of T cells were analyzed. (I) Representative confocal images of T cells (upper panel, from G; lower panel, from $\mathrm{H}$ ) at the indicated time points. iRGD-anti-CD3 was detected with Alexa Fluor 488-anti-His tag antibody, green; nucleus, blue. Scale bar, $5 \mu \mathrm{m}$. PBMC, peripheral blood mononuclear cell; VH, heavy chain variable domain; VL, light chain variable domain.

cytoplasmic compartment at 36 hours, with a concomitant decrease in cell surface signal (figure 1I). Meanwhile, iRGD-anti-CD3 underwent dissociation from bound $\mathrm{T}$ cells, which could be quantified as the reduction in overall fluorescence intensity (online supplemental figure S2B).

\section{iRGD-anti-CD3 modification promoted T-cell infiltration in tumor spheroids and xenograft mouse tumors}

Considering monolayer cell cultures do not allow infiltration studies, we employed MCS from human gastric cell line HGC27. ${ }^{12}$ As shown in figure 2A, PBMCs alone or modified with $\mathrm{CG}_{7} \mathrm{C}$-anti-CD3 were constrained to 
A

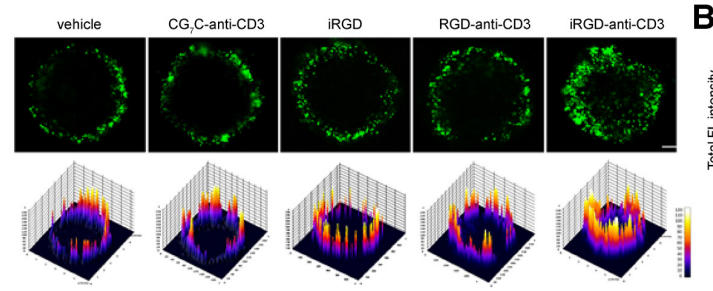

C

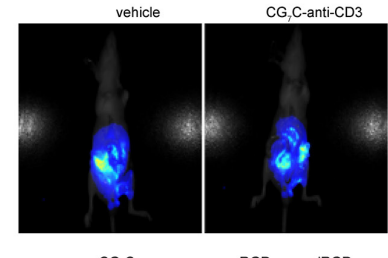

D
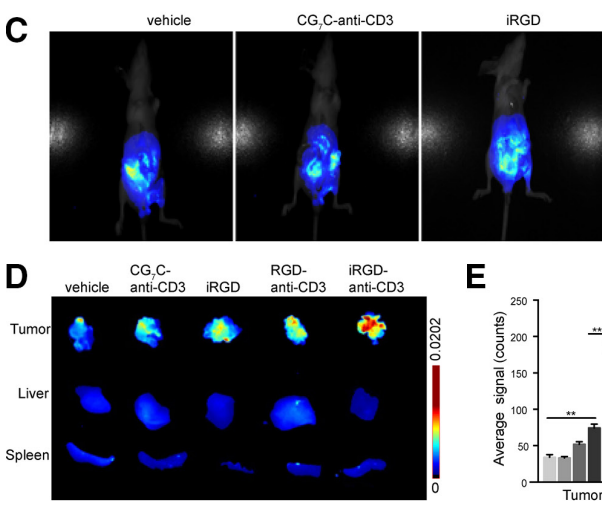

E

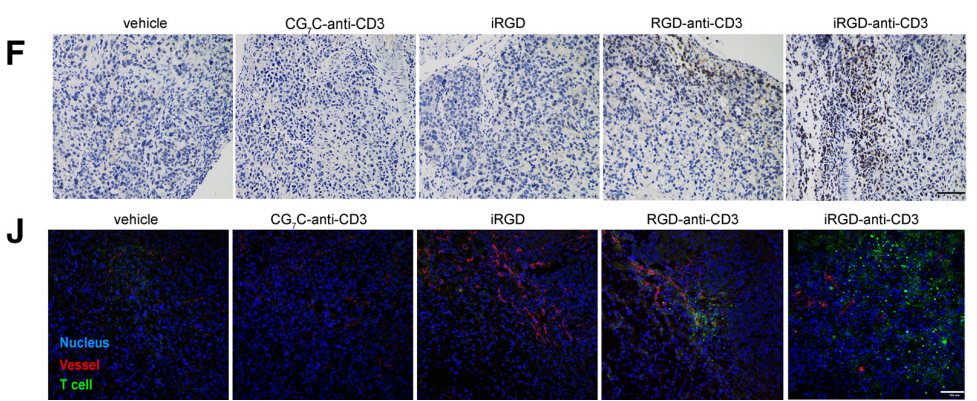

G

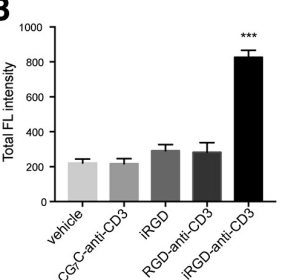

iRGD-anti-CD3

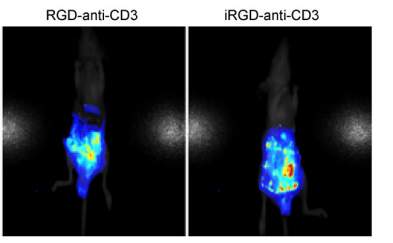

${ }^{250} 7$ vehicle

- $\mathrm{CG}_{7} \mathrm{C}-$ anti-CD3

iRGD

RGD-anti-CD3

iRGD-anti-CD3

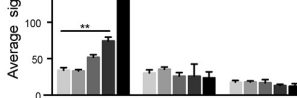

RGD-anti-CD3
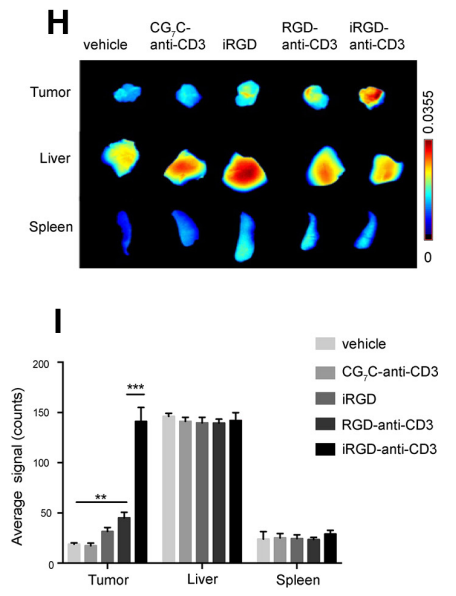

Figure 2 iRGD-anti-CD3 enhanced T-cell penetration in HGC27 spheroids and tumor nodules in vivo. (A) Confocal microscopy images (upper panel) and surface plot images (lower panel) showing penetration of CFSE-labeled PBMCs in MCSs. Magnification, $\times 200$; scale bar, $50 \mu \mathrm{m}$. (B) Mean CFSE fluorescence intensity (FL) of MCSs in (A). (C) In vivo near-infrared imaging of intraperitoneal tumor-bearing mice at 6 hours after intraperitoneal injection of $T$ cells. (D) Ex vivo images of tumors, livers and spleens collected at 6 hours after injection. (E) Semiquantification of T-cell signal in tumors, livers and spleens. (F) Immunohistochemistry analysis of tumor sections at 6 hours after injection. T cells were stained with an anti-CD3 mAb. (G) In vivo imaging of subcutaneous tumor-bearing mice at different time points after intravenous injection of $\mathrm{T}$ cells. Magenta dashed lines indicate tumors. (H) Ex vivo images of tumors, livers and spleens 24 hours after intravenous injection. (I) Semiquantification of T-cell signal in tumors, livers and spleens. (J) Immunofluorescence analysis of tumor sections at 24 hours after injection. T cells were labeled with CFSE (green); tumor blood vessels were stained with a Cy3-anti-CD31 antibody (red); nucleus, blue. Results are presented as mean fluorescence intensity $\pm S E M, n=3$. Student's t-test; ${ }^{* *} p<0.01,{ }^{* \star *} p<0.001$. Scale bars, $100 \mu \mathrm{m}$. MCS, multicellular spheroid; PBMC, peripheral blood mononuclear cell.

the periphery of the MCSs where only weak signals were detected. Free iRGD and RGD-anti-CD3 slightly improved PBMC penetration. In comparison, iRGD-anti-CD3 modification enabled PBMCs to actively penetrate deep into the spheroids, and a fraction of them reached the center. The fluorescence intensity was consistently increased in the iRGD-anti-CD3 group (figure 2B). No significant difference in penetration was found between $T$ cells modified with iRGD-anti-CD3 and DSPE-PEG-iRGD (online supplemental figure S3).

We then investigated whether iRGD-anti-CD3 could improve T-cell infiltration in the gastric cancer peritoneal metastasis mouse model verified in our previous work. ${ }^{12}$ As shown in figure $2 \mathrm{C}-\mathrm{E}$ and online supplemental figure S4, iRGD-anti-CD3-modified T cells effectively accumulated in peritoneal tumors, as evidenced by significantly $(p<0.001)$ stronger fluorescence signal in tumor nodules and comparatively less signal in the liver. DSPE-PEGiRGD modification increased T-cell accumulation to a comparable extent. RGD-anti-CD3 had a modest effect on T-cell accumulation into tumors, and only a minimal signal was observed in the other three groups. None of the experimental groups increased T-cell signal in other main organs (online supplemental figure S5). We further compared the penetration capability of $\mathrm{T}$ cells in tumor parenchyma. Neither $\mathrm{T}$ cells alone nor $\mathrm{T}$ cells modified with $\mathrm{CG}_{7} \mathrm{C}$-anti-CD3 or free iRGD could enter tumor nodules. $\mathrm{T}$ cells in the RGD-anti-CD3 group were restricted to the tumor periphery. Nevertheless, T cells modified with iRGD-anti-CD3, as well as DSPE-PEG-iRGD, were found extensively dispersed within tumor tissues (online supplemental S4D). 
We also demonstrated homing and penetration of iRGD-anti-CD3-modified T cells in a subcutaneous tumor model. As shown in figure $2 \mathrm{G}$, $\mathrm{T}$ cells alone or modified with $\mathrm{CG}_{7} \mathrm{C}$-anti-CD3 were primarily retained in the liver, with only a faint signal detected in tumor regions. Free iRGD and RGD-anti-CD3 were slightly effective in improving T-cell homing. Strikingly, iRGD-anti-CD3modified T cells exhibited strong signal in tumors which peaked at 24 hours, maintained up to 48 hours and then declined until 72 hours after treatment. Tumors were resected at 24 hours and measured ex vivo (figure $2 \mathrm{H}, \mathrm{I}$ ). In line with the whole-body imaging, we indeed found the most T-cell accumulation in the iRGD-anti-CD3 group, which was more than sevenfold and threefold that of the vehicle control and RGD-anti-CD3 groups, respectively. Immunofluorescence analyses were preformed to further evaluate T-cell penetration (figure 2J). iRGD-anti-CD3 modification mediated extensive T-cell distribution in areas far from tumor vessels, while $\mathrm{T}$ cells in the RGDanti-CD3 group were trapped around blood vessels. The results demonstrated that iRGD-anti-CD3 modification endowed $\mathrm{T}$ cells with a superior infiltration capacity in MCSs and tumor tissues in vivo.

\section{iRGD-anti-CD3 induced tumor-specific T-cell activation and cytotoxicity}

Besides promoting T-cell infiltration, we further tested the ability of iRGD-anti-CD3 to activate T cells. In the presence of target tumor cells, $\mathrm{CD}^{+}$and $\mathrm{CD}^{+} \mathrm{T}$ cells within PBMCs modified with iRGD-anti-CD3 significantly elevated surface expression of CD69 and CD25, the respective early and late activation markers (figure 3A,B). T-cell activation was also reflected by favorable cytokine secretion towards a Th1 immune response including IFN- $\gamma$ and tumor necrosis factor alpha in culture supernatants (figure 3C). Along with activation, iRGD-anti-CD3 mediated potent T-cell cytotoxicity in an E:T ratiodependent manner (figure 3D and online supplemental figure S6). Tumor lysis was observed even at an E:T ratio down to 5:1. Notably, these aforementioned T-cell activities required interactions with target tumor cells, as RGDanti-CD3 functioned similarly but $\mathrm{CG}_{7} \mathrm{C}$-anti-CD3 did not lead to any activation or lysis. The results demonstrated that iRGD-anti-CD3 induced tumor-specific T-cell activation and cytotoxicity, which was strictly dependent on the recognition of iRGD receptors.

We then explored the killing capacity of iRGD-antiCD3-modified T cells in MCSs (figure 3E,F, and online supplemental figures $\mathrm{S} 7$ and $\mathrm{S} 8$ ). Tumor cells in the vehicle control and $\mathrm{CG}_{7} \mathrm{C}$-anti-CD3 groups were all mostly alive. free iRGD and RGD-anti-CD3 led to a limited amount of cell death. In contrast, $T$ cells modified with iRGD-anti-CD3 displayed more robust killing efficacy, even compared with those with DSPE-PEG-iRGD. The spherical shape and compactness of MCSs were destroyed
A
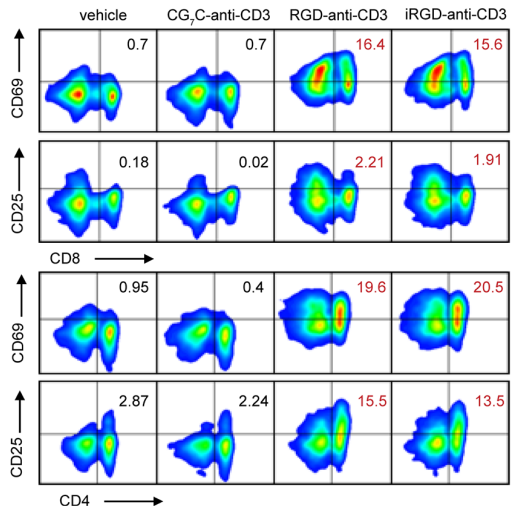

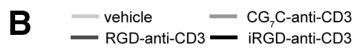

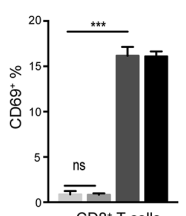

$\mathrm{CD}^{+} \mathrm{T}$ cells
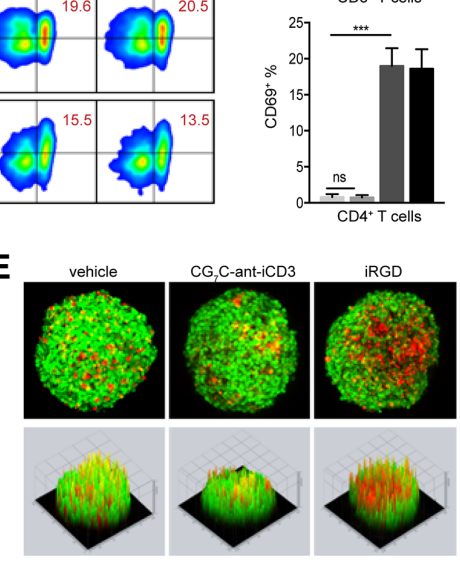

iRGD

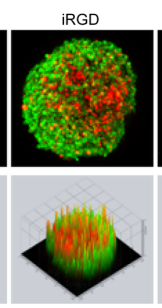

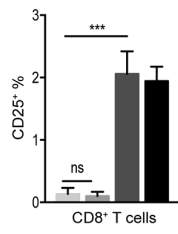
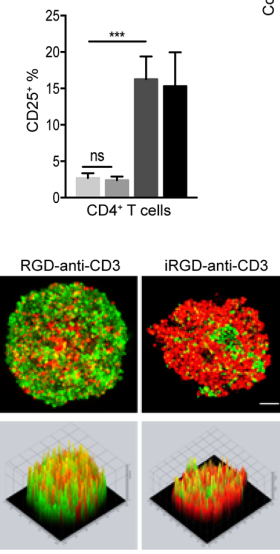

C
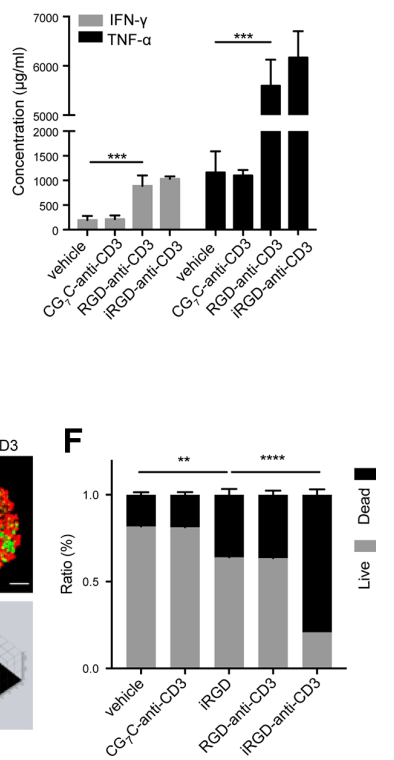

Figure 3 iRGD-anti-CD3 promoted T-cell activation and cytotoxicity. (A) Flow cytometry results and (B) quantification of CD69 and CD25 expression on $\mathrm{CD}^{+}$and $\mathrm{CD} 4^{+}$T-cell subsets. (C) Assessment of IFN- $\gamma$ and TNF- $\alpha$ in coculture supernatants. (D) Lysis of monolayer tumor cells at indicated E:T ratios. (E) Confocal microscopy images (upper panel) and surface display (lower panel) of live/dead viability assays in MCSs. Live cells were stained with calcein acetoxymethyl ester (AM) (green) and dead cells with EthD-1 (red); magnification, $\times 200$; scale bar, $50 \mu \mathrm{m}$. (F) Live/dead cell quantification in MCSs. Data are represented as mean \pm SEM; $n=5$. Student's t-test; ${ }^{* \star} p<0.01,{ }^{\star \star \star} p<0.001,{ }^{* \star \star \star} p<0.0001 . C_{7} C-, G_{7} C$-anti-CD3; RGD-, RGD-anti-CD3; iRGD, iRGD-anti-CD3. E:T, effector-to-target; IFN- $\gamma$, interferon gamma; MCS, multicellular spheroid; TNF- $\alpha$, tumor necrosis factor alpha. 
and their viability markedly dropped to approximately $20 \%$. These results provided initial evidence that iRGDanti-CD3 modification promoted both T-cell infiltration and activation, a prerequisite for antitumor efficacy in vivo.

\section{Combining iRGD-anti-CD3 modification with PD-1 blockade potently inhibited tumor growth in xenograft mouse models}

Inhibitory molecules such as PD-1/PD-L1 could be upregulated as resistance to BiTE treatment, and the addition of immune checkpoint inhibitors (ICIs) has been demonstrated to partially restore BiTEs activity. ${ }^{17}{ }^{18}$ In this study, staining of tumors treated with iRGD-anti-CD3-modified T cells showed a higher degree of PD-L1 and PD-1 expressions, suggesting there might be potential synergistic effects with PD-1 blockade (figure 4A,B).

We first evaluated antitumor effects in mice bearing disseminated peritoneal tumors (online supplemental figure S9A). Bioluminescence imaging of luciferase-tagged MKN45 was used to allow dynamic quantification of tumor cells throughout the entire abdominal cavity (figure 4C-F and online supplemental figure S9B). All mice in the first five groups showed rapid tumor growth and developed massive tumor burdens and ascitic fluids; free iRGD and RGD-anti-CD3 only slightly delayed tumor growth, and PD-1 blockade alone was not effective for tumor control. In contrast, prominent tumor inhibition and prolonged survival were achieved with iRGD-anti-CD3-modified T cells. Importantly, the addition of PD-1 blockade provided greater antitumor efficacy, as evidenced by minimal tumor burden persisting until day 28, 9 days after treatment was stopped. The average signal in the combination group showed an over 3-fold and nearly 10-fold reduction compared with the iRGD-anti-CD3 group and the other control groups, respectively. The addition of PD-1 blockade likewise provided superior survival benefits (online supplemental figure S9C). However, T cells modified with DSPEPEG-iRGD showed a weaker blocking effect on tumor growth, as is the case with anti-PD-1 combination (online supplemental figure S9D). We then assessed the contribution of $\mathrm{CD} 4$ and $\mathrm{CD} 8 \mathrm{~T}$ cells to antitumor responses of the combination therapy. As shown in figure $4 \mathrm{G}$ and online supplemental figure S10, CD8 depletion mostly abrogated growth inhibition induced by combing iRGD-anti-CD3 and PD-1 blockade, and CD4 depletion showed a modest effect on growth control. These results suggested that both CD4 and CD8 T cells drive the antitumor effect of the combination therapy. No formulations caused significant systemic toxicity as assessed by H\&E staining, suggesting a favorable safety profile of iRGD-anti-CD3 modification plus PD-1 blockade (online supplemental figure S11).

The merits of combination therapy were further investigated in the MGC803 peritoneal metastatic mouse model (figure $4 \mathrm{H}, \mathrm{I}$, and online supplemental figure S12). Mice treated with iRGD-anti-CD3-modified T cells exhibited remarkably smaller tumor weights compared with vehicle control and PD-1 blockade alone. The combination group displayed a trend of less tumor burden, although the difference was not statistically significant. Moreover, as receptors for iRGD were ubiquitously upregulated in tumor endothelial cells and various other tumor cells, we hypothesized that the combination therapy might also benefit other types of solid tumors. Thus, we used another mouse model with LOVO peritoneal metastatic colon cancer. At the final observation point, mice in the combination group had the mildest abdominal distension (online supplemental figure S13A,B). Analysis of both tumor weight and ascites volume confirmed that combining iRGD-anti-CD3 modification with PD-1 blockade was the most effective therapy (figure 4J,K, and online supplemental figure S13C,D).

We next studied antitumor efficacy in mice bearing subcutaneous tumors. iRGD-anti-CD3-modified $\mathrm{T}$ cells suppressed tumor growth to a certain extent, while combination with PD-1 blockade performed the best (figure 4L). Whereas $50 \%$ of the mice in the combination group were still alive after 55 days, there was little survival in the iRGDanti-CD3 group and $100 \%$ mortality in the other control groups (figure $4 \mathrm{M}$ ). H\&E staining showed no obvious tissue toxicity in any of the experimental groups (online supplemental figure S14). Overall, these results indicated that combining iRGD-anti-CD3 modification with PD-1 blockade produced far-reaching antitumor efficacy.

\section{iRGD-anti-CD3 induced a vesicular channel for transendothelial T-cell migration}

In our previous work, binding of iRGD to NRP-1 has been found to facilitate $\mathrm{T}$ cell migration by opening endothelial junctions through tyrosine phosphorylation of vascular endothelial (VE)-cadherin. ${ }^{12}$ However, many studies have revealed that, besides this paracellular route, a vesicular transport pathway in the endothelial cytoplasm termed vesiculovacuolar organelles (VVOs) also participates in leukocyte extravasation during inflammation. ${ }^{19-21}$ Therefore, we hypothesized that VVOs might have a vital role in iRGD-mediated T-cell infiltration. TEM was used for ultrastructural analysis of tumor vessels. We found that, at 24 hours after injection, iRGD-anti-CD3 induced the enrichment of single grape-like vesicles and fusion of multiple vesicles within the cytoplasm of endothelial cells (figure 5A). Quantitative analysis of vesicle density showed that iRGDanti-CD3 resulted in nearly 10 -fold more vesicles than the vehicle control and RGD-anti-CD3 (figure 5B). The results supported that binding of iRGD-anti-CD3 to NRP-1 could initiate VVO formation to facilitate T-cell migration through the body of endothelial cells.

\section{DISCUSSION}

Efficient T-cell infiltration into tumors is fundamental to the success of adoptive cell immunotherapy. The process of T-cell trafficking to the tumor site comprises three classical steps: rolling, adhesion, and transmigration. ${ }^{20}$ In each of these steps, tumors can create different barriers to disrupt T-cell entry, such as downregulated expression of chemokines and adhesion molecules, aberrant vessel leakiness, and immune suppressive microenvironment. ${ }^{322}$ For enhancing 

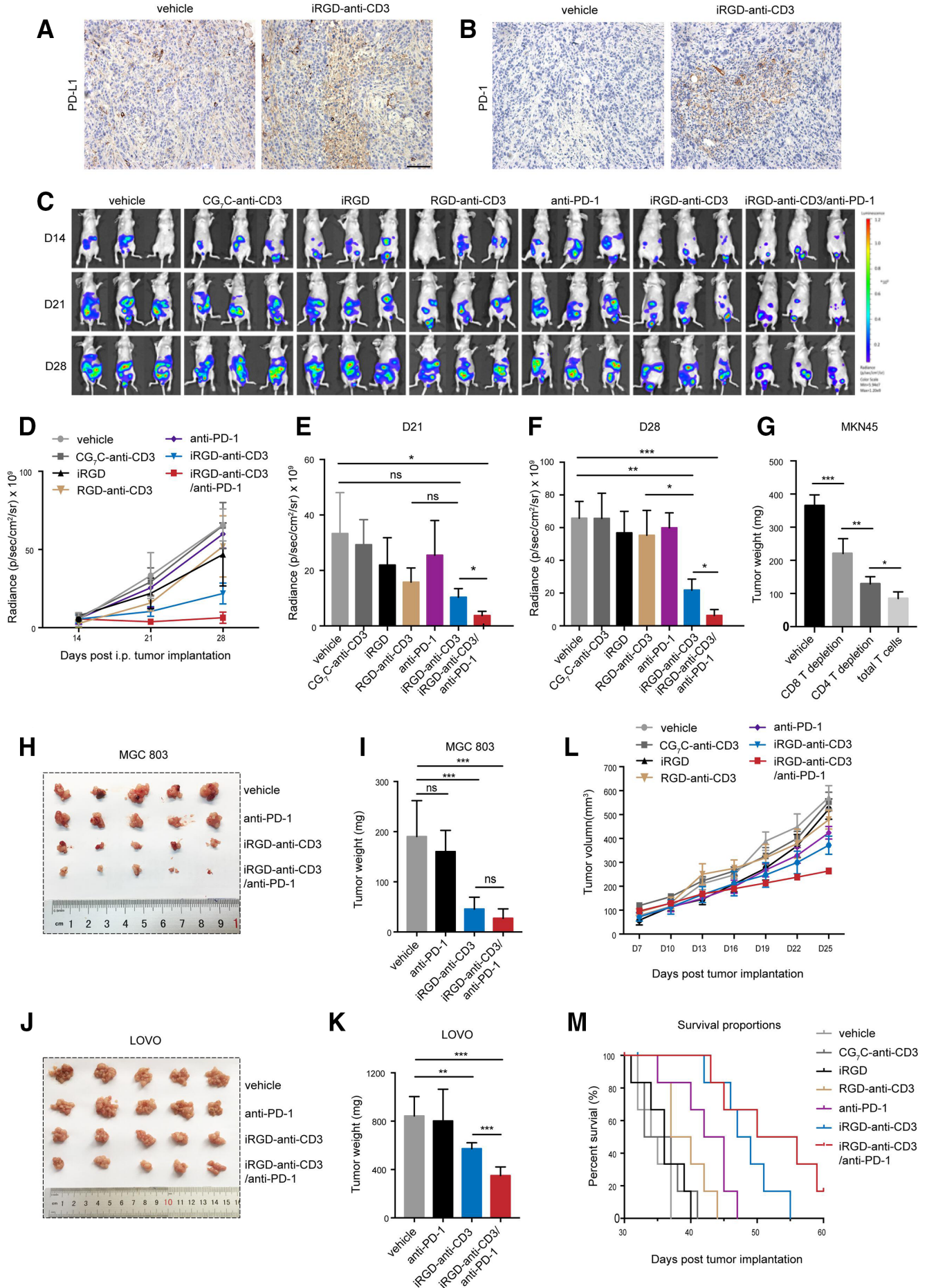

Figure 4 Combination of iRGD-anti-CD3-modified T cells with PD-1 blockade effectively controlled tumor growth. (A) Immunohistochemistry analysis of PD-L1 and (B) PD-1 expression in peritoneal tumors. Scale bar, $100 \mu \mathrm{m}$. (C-F) MKN45-luc peritoneal metastasis tumor model, $\mathrm{n}=3$. Two weeks after tumor implantation (day 15 ), $1 \times 10^{7} \mathrm{~T}$ cells modified with $100 \mu \mathrm{g}$ iRGDanti-CD3 or control reagents or codelivered with $3 \mu \mathrm{g}$ free iRGD were given intraperitoneally every other day for 5 days. PD-1 blockade of $250 \mu \mathrm{g}$ was given intraperitoneally every other day for a total of three doses. (C) Anterior bioluminescence images of tumor burden at days 14 (before treatment), 21 and 28 after tumor inoculation. (D) Curves of tumor signal at three indicated timepoints during the treatment course. (E) Tumor signal quantification at 21 days and $(F) 28$ days after tumor inoculation. (G) $1 \times 10^{7}$ total T cells or with CD8/CD4 depletion were modified with $100 \mu \mathrm{g}$ iRGD-anti-CD3 and then injected into mice bearing disseminated MKN45 peritoneal tumors every other day three times. PD-1 blockade of $250 \mu \mathrm{g}$ was given intraperitoneally every other day for three injections. Tumors were harvested after 2 weeks of treatment and weighed. $(\mathrm{H})$ Tumor nodules in all treatment groups were photographed and (I) weighed in the MGC803 peritoneal metastatic tumor model. (J) Representative images and $(K)$ weight of excised tumors in LOVO peritoneal metastasis tumor model. (L) Tumor growth profiles and (M) survival analysis in MKN45 subcutaneous mouse model, $n=6$. Data are analyzed with Student's t-test unless specified, and are represented as mean \pm SEM; ${ }^{*} p<0.05,{ }^{* *} p<0.01,{ }^{* * *} p<0.001$. ns, not significant. 
A

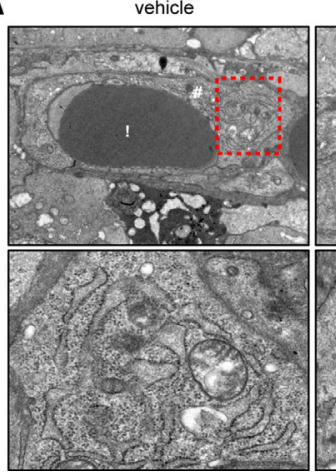

RGD-anti-CD3

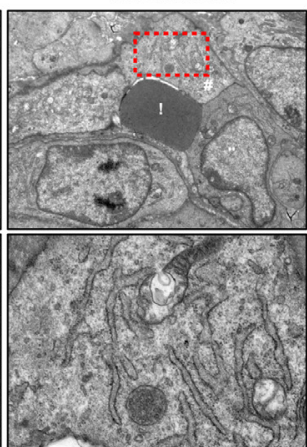

iRGD-anti-CD3

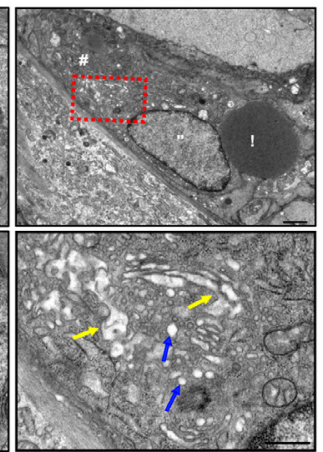

B

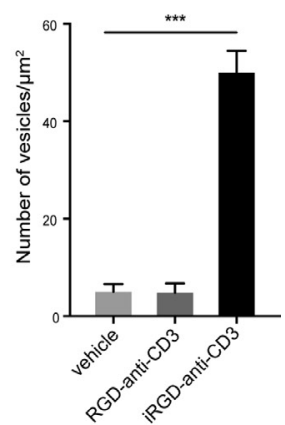

Figure 5 Underlying mechanisms of T-cell infiltration mediated by iRGD-anti-CD3. (A) Representative TEM images of tumor vessels at 24 hours after injection of PBS (left), RGD-anti-CD3 (middle) and iRGD-anti-CD3 (right). Structures in red dashed box are shown with higher magnification in the corresponding panels below. Blue arrows: single vesicles, yellow arrows: fused vesicles. Scale bars, $1 \mu \mathrm{m}$ (upper panels), $0.5 \mu \mathrm{m}$ (under panels). (B) vesicle density shown as the number of vesicles per $1 \mu \mathrm{m}^{2}$ of cytoplasmic area. data are represented as mean $\pm S E M ; n=3$. Student's t-test, ${ }^{\star \star \star} p<0.001$. C, cytoplasm of the endothelial cell; $\mathrm{N}$, nucleus of the endothelial cell; PBS, phosphate-buffered saline; R, red blood cell; TEM, transmission electron microscopy.

T-cell infiltration, a number of strategies including transducing $\mathrm{T}$ cells with chemokine receptors, intratumoral injection of IFN- $\gamma$ to induce chemokine production, and combinations with anti-angiogenesis agents or ICIs have been investigated. ${ }^{23-25}$ These therapies required complex genetic or technological manipulation or may increase treatment-related adverse effects. Here we provided an innovative and efficient strategy by functionalizing $\mathrm{T}$ cells with the tumor-penetrating peptide iRGD. iRGD facilitates T-cell infiltration in two aspects. First, iRGD binds to elevated integrins which support firm T-cell adhesion, and therefore increases accumulation of modified $\mathrm{T}$ cells in tumor vessels. ${ }^{1920}$ Then by binding to the second receptor NRP-1, iRGD initiates two distinct pathways for T cells to migrate through blood walls. One pathway that opens endothelial junctions through the tyrosine phosphorylation of VE-cadherin has been previously demonstrated. ${ }^{12}$ Here we reported another equally important but less often concerned mechanism using TEM. Vesicular channels composed of VVOs clusters were found within the cytoplasm of endothelial cells by which T cells migrated directly through the body of the endothelium. These VVOs usually occur in 'thin' parts of the endothelium so that T cells may extravasate with less distance and quickly reach the tumor parenchyma. $^{20}$

iRGD can be modified on T-cell surface in different ways. DSPE-PEG was used in our previous work; DSPE was inserted into cell membranes through hydrophobic interaction, while PEG was connected to iRGD through Michael addition-reaction, thereby anchoring iRGD on the T-cell surface. Although this method significantly enhanced T-cell infiltration capability, we aimed to find an optimal modification approach which would further improve killing activity of infiltrating $\mathrm{T}$ cells and simultaneously avoid disruption that DSPE-PEG might cause on cell membranes. The anti-CD3 scFv is such an ideal connector as it triggers activation signaling on binding to the CD $3 \varepsilon$ on the T-cell surface. Its efficacy and safety have been fully demonstrated by BiTE therapy. Besides the anti-CD19 BiTE approved for the treatment of hematological malignancies, at least five BiTEs are being tested in phase I clinical trials for solid tumor indications and have shown promising antitumor effects with manageable safety profiles. ${ }^{152627}$ Therefore, we genetically fused iRGD with the anti-CD3 scFv and generated a novel recombinant antibody iRGD-anti-CD3. T cells modified with iRGD-anti-CD3 showed four to seven times more accumulation and penetration in both MCSs and in vivo tumor nodules than $\mathrm{T}$ cells alone. More importantly, iRGD-anti-CD3 induced T-cell activation evidenced by upregulation of activation markers, Th1-cytokines, and cytotoxicity against monolayer tumor cells and MCSs. Enhanced penetration, together with activation, eventually translated into potent antitumor efficacy of T cells modified with iRGD-anti-CD3, as demonstrated in several xenograft mouse models.

In addition to RGD-anti-CD3 for highlighting the importance of NRP-1 pathway to T-cell penetration, we also included two other control reagents $\mathrm{CG}_{7} \mathrm{C}$-anti-CD3 and free iRGD. $\mathrm{CG}_{7} \mathrm{C}$-anti-CD3, which recognizes neither tumor blood vessels nor tumor tissues, had no effects on activation or antitumor efficacy of T cells. This supports that monovalent CD3 binding is not enough to trigger T-cell signaling due to low affinity, unless the CD3 scFv is presented to T cells in a multivalent fashion by target cells. ${ }^{28}{ }^{29}$ Therefore, T-cell lysis could be exclusively redirected to tumor sites with aberrant expression of iRGD receptors while sparing normal tissues, as is the case with specific T-cell responses. iRGD has been widely reported to enhance tumor-specific delivery of anticancer drugs, and this effect does not require drugs to be conjugated to the peptide. ${ }^{10}{ }^{30}$ However, in our study, $\mathrm{T}$ cells coadministrated with free iRGD showed minimal improvements in tumor penetration compared with those modified with iRGD-anti-CD3, although the same amount of iRGD was given in both groups. This may be attributed to the limited opening time of migration routes triggered by free iRGD (within $15 \mathrm{~min}$ ) and the different trafficking times between iRGD and T cells into tumors. In contrast, in the iRGD-anti-CD3 group, the opening time of migration 
routes could be extended to as long as 24 hours. Moreover, immobilizing iRGD on surface of $\mathrm{T}$ cells allows them to reach tumor sites at the same time.

Still, combination with modified T cells and PD-1 blockade did not inhibit tumor growth in the subcutaneous tumor model to a comparable extent as in peritoneal metastasis models. At the end of tumor growth profiling, we found that the combination therapy caused necrosis inside subcutaneous tumors, and this antitumor effect could not be reflected by measuring tumor volumes. In vivo functional imaging approaches such as bioluminescence imaging may be more appropriate to accurately evaluate tumor burdens and treatment effects. Moreover, necrosis could lead to reduced tumor vascular supply, and hence trafficking of modified $\mathrm{T}$ cells, which greatly restricted T-cell efficacy against residual tumor tissues. Combining with other therapeutic strategies might be perceived as pivotal to further increase antitumor potency in subcutaneous models, and we are currently investigating the combination of local radiotherapy.

Taken together, we developed a novel bifunctional agent, iRGD-anti-CD3. T cells modified with iRGD-anti-CD3 exhibited both enhanced penetration capability and killing activity in MCSs and xenograft tumor models. Moreover, the combination with PD-1 blockade revealed superior antitumor effects. This combination strategy holds great potential to improve the efficacy of adoptive cell immunotherapy in multiple solid tumor types.

Acknowledgements We thank Professor Yifan Hu (Nanjing Medical University, Nanjing, China) for his technical support in using the confocal microscope.

Contributors SZ conducted experiments, wrote the main manuscript text, and prepared figures. SZ, FM, BL, and JW conceived and designed all experiments. $H Q$, ND, and HS provided protocols and tools for research. SD and MZ assisted in conducting the experiments and preparing the figures. JW and LW assisted in manuscript preparation. XY provided help in the 3D modeling software. BL and JW coordinated the research and verified the results. All authors reviewed the manuscript.

Funding This work was funded by grants from the National Major Projects for 'Major New Drugs Innovation and Development' (number 2019ZX09301150), the National Natural Science Foundation of China (number 81930080), the Fundamental Research Funds for the Central Universities (number 14380497), the Natural Science Foundation of Jiangsu Province (number BK20181115) and the Fund for Distinguished Young Scholars of Jiangsu Province (number BK20190001).

Competing interests None declared.

Patient consent for publication Not required.

Ethics approval The blood collection procedure was carried out in accordance with the guidelines verified and approved by the ethics committee of Drum Tower Hospital. All donors signed an informed consent for scientific research statement. All animal procedures were carried out in compliance with the guidelines set by the Animal Care Committee at Drum Tower Hospital (Nanjing, China). The ethics committee of Drum Tower Hospital approved all experiments in this study.

Provenance and peer review Not commissioned; externally peer reviewed.

Data availability statement Data are available upon reasonable request. All data relevant to the study are included in the article or uploaded as supplementary information. The data that support the findings of this study are available from the corresponding author (weijia01627@hotmail.com) upon reasonable request.

Supplemental material This content has been supplied by the author(s). It has not been vetted by BMJ Publishing Group Limited (BMJ) and may not have been peer-reviewed. Any opinions or recommendations discussed are solely those of the author(s) and are not endorsed by BMJ. BMJ disclaims all liability and responsibility arising from any reliance placed on the content. Where the content includes any translated material, BMJ does not warrant the accuracy and reliability of the translations (including but not limited to local regulations, clinical guidelines, terminology, drug names and drug dosages), and is not responsible for any error and/or omissions arising from translation and adaptation or otherwise.

Open access This is an open access article distributed in accordance with the Creative Commons Attribution Non Commercial (CC BY-NC 4.0) license, which permits others to distribute, remix, adapt, build upon this work non-commercially, and license their derivative works on different terms, provided the original work is properly cited, appropriate credit is given, any changes made indicated, and the use is non-commercial. See http://creativecommons.org/licenses/by-nc/4.0/.

\section{ORCID iD}

Shujuan Zhou http://orcid.org/0000-0002-2479-9246

\section{REFERENCES}

1 Melero I, Rouzaut A, Motz GT, et al. T-Cell and NK-cell infiltration into solid tumors: a key limiting factor for efficacious cancer immunotherapy. Cancer Discov 2014;4:522-6.

2 Fridman WH, Pagès F, Sautès-Fridman C, et al. The immune contexture in human tumours: impact on clinical outcome. Nat Rev Cancer 2012;12:298-306.

3 Slaney CY, Kershaw MH, Darcy PK. Trafficking of T cells into tumors. Cancer Res 2014;74:7168-74.

4 van der Woude LL, Gorris MAJ, Halilovic A, et al. Migrating into the tumor: a roadmap for T cells. Trends Cancer 2017;3:797-808.

5 Carmeliet P, Jain RK. Principles and mechanisms of vessel normalization for cancer and other angiogenic diseases. Nat Rev Drug Discov 2011;10:417-27.

6 Moon EK, Carpenito C, Sun J, et al. Expression of a functional CCR2 receptor enhances tumor localization and tumor eradication by retargeted human $T$ cells expressing a mesothelin-specific chimeric antibody receptor. Clin Cancer Res 2011;17:4719-30.

7 Sugahara KN, Teesalu T, Karmali PP, et al. Tissue-penetrating delivery of compounds and nanoparticles into tumors. Cancer Cell 2009;16:510-20.

8 Liu X, Lin P, Perrett I, et al. Tumor-penetrating peptide enhances transcytosis of silicasome-based chemotherapy for pancreatic cancer. J Clin Invest 2017;127:2007-18.

9 Wang Y, Xie Y, Li J, et al. Tumor-penetrating nanoparticles for enhanced anticancer activity of combined photodynamic and hypoxia-activated therapy. ACS Nano 2017;11:2227-38.

10 Sugahara KN, Scodeller P, Braun GB, et al. A tumor-penetrating peptide enhances circulation-independent targeting of peritoneal carcinomatosis. J Control Release 2015;212:59-69.

11 Teesalu T, Sugahara KN, Kotamraju VR, et al. C-end rule peptides mediate neuropilin-1-dependent cell, vascular, and tissue penetration. Proc Natl Acad Sci U S A 2009;106:16157-62.

12 Ding N, Zou Z, Sha H, et al. iRGD synergizes with PD-1 knockout immunotherapy by enhancing lymphocyte infiltration in gastric cancer. Nat Commun 2019;10:1336.

13 Che J, Okeke Cl, Hu Z-B, et al. DSPE-PEG: a distinctive component in drug delivery system. Curr Pharm Des 2015;21:1598-605.

14 Klinger M, Benjamin J, Kischel R, et al. Harnessing T cells to fight cancer with BiTE $尺$ antibody constructs--past developments and future directions. Immunol Rev 2016;270:193-208.

15 Labrijn AF, Janmaat ML, Reichert JM, et al. Bispecific antibodies: a mechanistic review of the pipeline. Nat Rev Drug Discov 2019;18:585-608.

16 Huehls AM, Coupet TA, Sentman CL. Bispecific T-cell engagers for cancer immunotherapy. Immunol Cell Biol 2015;93:290-6.

17 Chang C-H, Wang Y, Li R, et al. Combination therapy with bispecific antibodies and PD-1 blockade enhances the antitumor potency of $T$ cells. Cancer Res 2017;77:5384-94.

18 Kobold S, Pantelyushin S, Rataj F, et al. Rationale for combining bispecific $T$ cell activating antibodies with checkpoint blockade for cancer therapy. Front Oncol 2018;8:285.

19 Nourshargh S, Hordijk PL, Sixt M. Breaching multiple barriers: leukocyte motility through venular walls and the interstitium. Nat Rev Mol Cell Biol 2010;11:366-78.

20 Ley K, Laudanna C, Cybulsky MI, et al. Getting to the site of inflammation: the leukocyte adhesion cascade updated. Nat Rev Immunol 2007;7:678-89.

21 Muller WA. Transendothelial migration: unifying principles from the endothelial perspective. Immunol Rev 2016;273:61-75.

22 Sackstein R, Schatton T, Barthel SR. T-Lymphocyte homing: an underappreciated yet critical hurdle for successful cancer immunotherapy. Lab Invest 2017;97:669-97. 
23 Mauldin IS, Wages NA, Stowman AM, et al. Intratumoral interferongamma increases chemokine production but fails to increase $T$ cell infiltration of human melanoma metastases. Cancer Immunol Immunother 2016:65:1189-99.

24 Chheda ZS, Sharma RK, Jala VR, et al. Chemoattractant receptors BLT1 and CXCR3 regulate antitumor immunity by facilitating CD8+ T cell migration into tumors. J Immunol 2016;197:2016-26.

25 Khan KA, Kerbel RS. Improving immunotherapy outcomes with anti-angiogenic treatments and vice versa. Nat Rev Clin Oncol 2018:15:310-24.

26 Pishvaian M, Morse MA, McDevitt $\mathrm{J}$, et al. Phase 1 dose escalation study of MEDI-565, a bispecific T-cell engager that targets human carcinoembryonic antigen, in patients with advanced gastrointestinal adenocarcinomas. Clin Colorectal Cancer 2016;15:345-51.
27 Fiedler WM, Ritter B, Seggewiss R, et al. Phase I safety and pharmacology study of the EpCAM/CD3-bispecific bite antibody MT110 in patients with metastatic colorectal, gastric, or lung cancer. JCO 2010;28:2573.

28 Brischwein K, Parr L, Pflanz S, et al. Strictly target cell-dependent activation of T cells by bispecific single-chain antibody constructs of the bite class. J Immunother 2007:30:798-807.

29 Dreier T, Lorenczewski G, Brandl C, et al. Extremely potent, rapid and costimulation-independent cytotoxic T-cell response against lymphoma cells catalyzed by a single-chain bispecific antibody. Int $J$ Cancer 2002;100:690-7.

30 Sugahara KN, Teesalu T, Karmali PP, Tambet T, Priya Prakash K, et al. Coadministration of a tumor-penetrating peptide enhances the efficacy of cancer drugs. Science 2010;328:1031-5. 